\title{
Study of supercapacitors with a double electrical layer based on activated carbon materials
}

\author{
I.M. Budzulyak ${ }^{1)}$, Z.D. Kovalyuk ${ }^{1)}$, F.V. Motsnyi ${ }^{2)}$, and V.B. Orletskyi ${ }^{1)}$ \\ 1) Chernivtsi Dept. of Institute for Problems of Materials Science, NAS of Ukraine, 5 I. Vilde, 58001 Chernivtsi, Ukraine \\ ${ }^{2)}$ Institute of Semiconductor Physics, NAS of Ukraine, 45 prospect Nauky, 03028 Kyiv, Ukraine
}

\begin{abstract}
It is proposed ecologically pure technology to obtain activated carbon. On the base of this carbon the supercapacitors were manufactured. Their characteristics were determined and compare with analogs obtained using ecologically dangerous materials.
\end{abstract}

Keywords: activated carbon; supercapacitor; ecologically pure technology.

Paper received 17.10.01; revised manuscript received 24.12.01; accepted for publication 05.03.02.

\section{Introduction}

Technological process for obtaining activated carbon (AC) with highly-developed surface $\left(>1000 \mathrm{~m}^{2} / \mathrm{g}\right.$ ) for manufacturing supercapacitor (SC) with a double electric layer is complex, often ecologically dangerous process $[1,2]$. Therefore, search of new possibilities to obtain AC that improve fabrication process and eliminate existing defects seems to be urgent. In the present work possibilities of obtaining AC in closed volume were studied when carbonization and activation of initial material take place without inert gas as a carrier of pore generator.

In contrast to traditional technologies, when flow of vapor and inert gas takes place at the same temperature, respectively, outflow this mass plus reaction products take place at another temperature, in our case, mass and heat transport are practically excluded. That allows to control effectively technological regime of obtaining AC.

Thus, the aim of the present work is to obtain activated carbon with developed surface area by ecologically pure technology, to manufacture supercapacitors on its base and to determine their characteristics.

\section{Results and discussion}

As an initial material for obtaining AC ecologically pure material, fruit pips (cherry, plum, cherry sweet) were used, while in majority technologies ecologically dangerous (especially at heating) organic compounds (formaldehyde, hydrocelluloze, sterol, etc) are used. As a pore generator $5 \div 20 \%$ water solution of $\mathrm{KOH}$ was used. Milled initial material was heated in vacuum with residual pressure less than $10^{2} \mathrm{~mm} \mathrm{Hg}$ and the temperature at which their full carbonization takes place. Then obtained material in mixture with $0 \div 20 \%$ water solution of $\mathrm{KOH}$ was heated in closed container up to $840 \div 880^{\circ} \mathrm{C}$ and kept up at this temperature during $70 \div 80 \mathrm{~min}$. In both cases heating was linear in time with the rate of $15 \div 20 \%$ min and cooling was in the turn-off furnace regime. As a consequence, carbonization of initial material and its activation take place. It is evident that with quantity of water or $\mathrm{KOH}$ solution one can easily control the vapor pressure, and in this way influence on process of pore generation and their sizes can be observed. At that temperature regime, the process is easily controlled since only pipe-bend of water vapor with decay products takes place. 
I.M. Budzulyak et al.: Study of supercapacitors with double electrical layer...

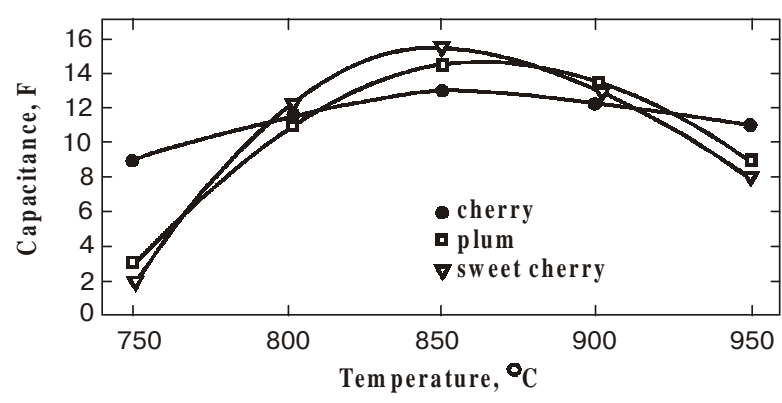

Fig. 1. Dependence of the capacitance of SC on the activation temperature of AC from which electrodes are formed.

Fig. 1 shows experimental dependence of the $\mathrm{SC}$ capacitance type in the body with the typesize ' 2525 ', formed on the base of obtained material, on temperature of activation. As it is seen from Fig. 1, optimal temperature for activation of preliminary carbonized initial material is $840 \div 880^{\circ} \mathrm{C}$ under other identical conditions. In this case, the internal resistance at mentioned temperatures lies in the range $0.08 \div 0.1 \Omega$. Achieved parameters of $\mathrm{SC}$ are not worse than the analogous ones formed on the base of AC from stirolvinilbenzol copolymer [3].

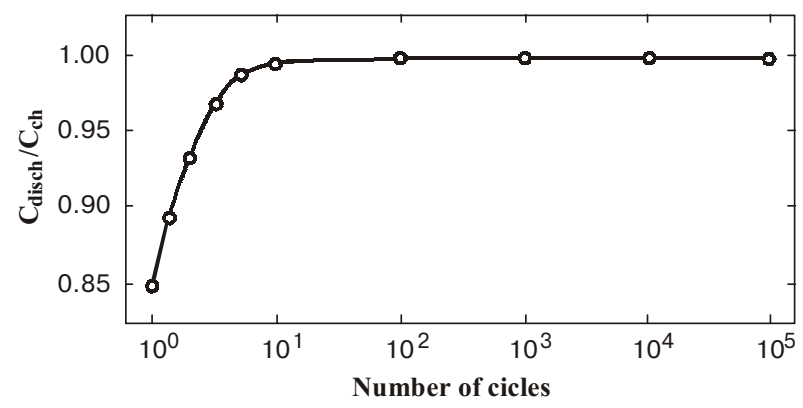

Fig. 2. The Coulomb efficiency of SC from obtained AC in the body typesize ' 2525 '.

Fig. 2 shows the Coulomb efficiency of SC formed on the base of obtained AC. As it is seen from Fig. 2, it is already after some cycles the Coulomb efficiency was stable at the level close to unity and was constant up to investigated $10^{5}$ cycles. This confirms that Faradey reactions in investigated SC are absent what is intrinsic to the ideal polarized electrods. Moreover, it is found that the typical charge-discharge curves at the charge current $0.05 \mathrm{~A}$ and at the discharge current $0.02 \mathrm{~A}$ are normal. This indicates that investigated capacitors are close to the classic ones.

Dependencies of obtained SC on the discharge current (Fig. 3) were also studied. Small differences in these dependencies as compared to SC formed using $\mathrm{AC}$ from stirolvinilbenzole copolymer were found.

Thus, the process of manufacturing AC is simplified as well as more controlled and ecologically secure. At the same time, operational characteristics of $\mathrm{SC}$ formed on its base is not worse than the analogous ones obtained using ecologically dangerous materials.

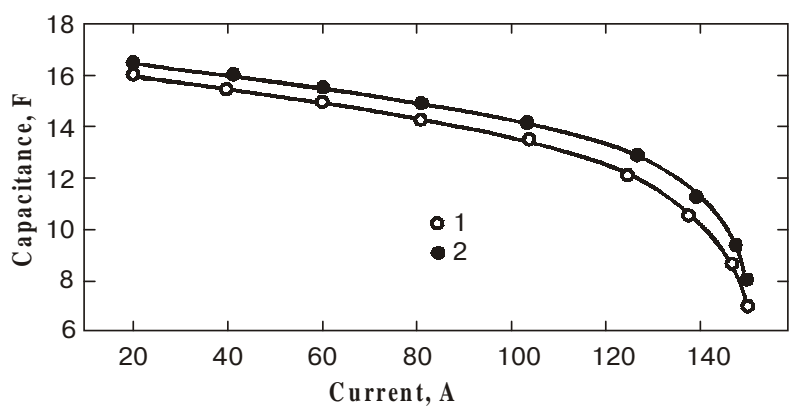

Fig. 3. Dependence of the capacitance of SC at typesize ' 2525 ' on the discharge current. 1- SC on the base of obtained AC, 2$\mathrm{SC}$ on the base of AC obtained from stirolvinilbenzole copolymer.

\section{Conclusions}

We proposed and realized a new technology for obtaining activated carbon. This technology uses ecologically pure materials. All technological process is also ecology secure and occurs in a closed volume without any inert gas. We obtained activated carbon with the developed surface area $900-1000 \mathrm{~m}^{2} / \mathrm{g}$ and manufactured supercapacitors on its base. Their capacities in the body with the typesize ' 2525 ' are about $10 \div 15 \mathrm{~F}$ and the internal resistances lie within the limits $0.06 \div 0.09 \Omega$.

\section{References}

1. G.Salitra, A.Soffer, L.Eliad et al., Carbon electrodes for double-layer capasitors//J.Electrochem.Soc., 147, p. 2486 (2000).

2. Electric double-layer capacitor and method to produce the same //US Patent No 4597028 (1984).

3. I.Grigorchak, Z.Kovalyuk, I.Koz'mik, Ionic capacitors (Preprint No 6 of Institute for Problems of Materials Science of NAS of Ukraine (1987), $32 \mathrm{p}$. 